\title{
On-pump versus off-pump coronary artery bypass surgery in high-risk patients: Operative results of a prospective randomized trial (on-off study)
}

\author{
Massimo G. Lemma, MD, PhD, FECTS, ${ }^{a}$ Enrico Coscioni, MD, ${ }^{\mathrm{b}}$ Francesco P. Tritto, MD, ${ }^{\mathrm{c}}$ \\ Paolo Centofanti, MD, ${ }^{\mathrm{d}}$ Corrado Fondacone, MD, ${ }^{\mathrm{e}}$ Andrea Salica, MD, ${ }^{\mathrm{f}}$ Agnese Rossi, MS, ${ }^{\mathrm{h}}$ Tiziana De \\ Santo, MS, ${ }^{\mathrm{h}}$ Giuseppe Di Benedetto, MD, ${ }^{\mathrm{b}}$ Luigi Piazza, MD, ${ }^{\mathrm{c}}$ Mauro Rinaldi, MD, ${ }^{\mathrm{d}}$ Antonio L. De Luca \\ Tupputi Schinosa, MD, ${ }^{\mathrm{e}}$ Ruggero De Paulis, MD, ${ }^{\mathrm{f}}$ Monica Contino, MD, ${ }^{\mathrm{a}}$ and Michele Genoni, $\mathrm{MD}^{\mathrm{g}}$
}

Objective: To analyze the risk reduction of cardiopulmonary bypass complications between on-pump and off-pump coronary artery bypass grafting in high-risk patients.

Methods: This multicenter, prospective, randomized, parallel trial enrolled patients for elective or urgent
isolated coronary artery bypass grafting with an additive European System for Cardiac Operative Risk
Evaluation of 6 or more. The patients in cardiogenic shock were excluded. The composite primary end
point included operative mortality, myocardial infarction, stroke, renal failure, reoperation for bleeding
and adult respiratory distress syndrome within 30 days after surgery. The total sample size was 693 pa-
tients, according to a scheduled interim analysis at 400 patients enrolled ( $\alpha$-spending $=0.029$, Pocock
method).

Results: A total of 411 patients were included in the interim analysis. Randomization assigned 203 patients to on-pump and 208 patients to off-pump treatment. Of the 411 patients, 24 crossed over; thus, 195 patients were actually treated on-pump and 216 off-pump. According to the intention to treat analysis, the rate of the composite primary end point was significantly lower (unadjusted $P=.009$, adjusted $P=.010$ ) in the off-pump group $(5.8 \%$ vs $13.3 \%)$. The risk of experiencing the primary end point was significantly greater for the on-pump group (unadjusted odds ratio, 2.51; 95\% confidence interval, $1.23-5.10 ; P=.011$; adjusted odds ratio, 3.07; $95 \%$ confidence interval, $1.32-7.14 ; P=.009)$.

Conclusions: Off-pump coronary artery bypass grafting reduces early mortality and morbidity in high-risk patients. (J Thorac Cardiovasc Surg 2012;143:625-31)

Earn CME credits at

http://cme.ctsnetjournals.org
From the Cardiovascular Surgery Department, "Luigi Sacco" University General Hospital, Milano, Italy; Cardiac Surgery Department, ${ }^{\text {b }}$ S. Giovanni di Dio e Ruggi D’Aragona Hospital, Salerno, Italy; Division of Cardiac Surgery, ${ }^{\mathrm{c}}$ San Sebastiano Hospital, Caserta, Italy; Department of Cardiac Surgery, ${ }^{\mathrm{d}}$ University of Medicine, S. Giovanni Battista Hospital, Turin, Italy; Department of Cardiac Surgery, ${ }^{\mathrm{e}}$ Policlinico Hospital, University of Bari, Bari, Italy; Division of Cardiac Surgery, ${ }^{\mathrm{f}}$ European Hospital, Rome, Italy; Cardiac Surgery Division, ${ }^{\mathrm{g}}$ City Hospital Triemli Zurich, Zurich, Switzerland; and Clinical Department, ${ }^{\text {h }}$ Medtronic Italy, Sesto San Giovanni, Italy.

This study was sponsored by Medtronic Italy.

Disclosures: Authors have nothing to disclose with regard to commercial support.

Read at the 91st Annual Meeting of The American Association for Thoracic Surgery, Philadelphia, Pennsylvania, May 7-11, 2011.

Received for publication April 12, 2011; revisions received Oct 19, 2011; accepted for publication Nov 8, 2011; available ahead of print Dec 12, 2011.

Address for reprints: Massimo G. Lemma, MD, PhD, FETCS, Department of Cardiovascular Surgery, Luigi Sacco University General Hospital, Via GB Grassi 74, Milan 20157 Italy (E-mail: lemma.massimo@hsacco.it).

$0022-5223 / \$ 36.00$

Copyright (c) 2012 by The American Association for Thoracic Surgery doi:10.1016/j.jtcvs.2011.11.011
Off-pump coronary artery bypass grafting (CABG) was introduced in the mid-1990s to reduce operative mortality and postoperative morbidity related to the use of cardiopulmonary bypass, including systemic inflammatory response, neurologic complications, global myocardial ischemia, renal failure, hemodynamic instability, and lung dysfunction. ${ }^{1,2}$ However, several prospective randomized clinical trials (RCTs) comparing off-pump CABG (OPCAB) and on-pump CABG (ECC) in lowrisk patients ${ }^{3,4}$ have not been able to demonstrate any significant reduction in mortality or major postoperative complications. More recently, some retrospective observational studies ${ }^{5,6}$ have shown that in high-risk patients, OPCAB can reduce operative mortality and postoperative morbidity compared with ECC, although 1 single-center RCT has shown no difference between the 2 techniques. $^{7}$

To further investigate the relative efficacy of ECC and OPCAB in high-risk patients (euroScore $\geq 6$ ), a RCT (onoff study, clinicaltrials.gov identifier NCT01290952) was designed to assess the operative, perioperative, and 1-year clinical outcomes in patients referred for isolated CABG. The present study reports the primary outcome results. 


$$
\begin{aligned}
& \text { Abbreviations and Acronyms } \\
& \text { ARDS = } \\
& \text { CABG = } \text { coronary artery bypass grafting } \\
& \text { ECC }= \text { on-pump coronary artery bypass } \\
& \text { grafting } \\
& \text { MI }= \text { myocardial infarction } \\
& \text { OPCAB = } \text { off-pump coronary artery bypass } \\
& \text { grafting } \\
& \text { RCT }= \text { randomized clinical trial }
\end{aligned}
$$

\section{MATERIALS AND METHODS Study Design}

The on-off study was a multicenter, prospective, randomized, parallel trial conducted from December 2006 through April 2010 at 7 Italian and 1 Swiss centers. The trial was designed by the principal investigators in cooperation with the sponsor (Medtronic Italy), approved by the institutional review board of each participating center, and assessed routinely by a monitoring board. All participants provided written informed consent. The protocol and consent form were consistent with the Declaration of Helsinki and all local regulations, as appropriate. The sponsor was involved in the collection and source verification of the data under the supervision of an independent clinical events committee composed of 3 physicians with relevant medical background in the field of cardiac surgery. Moreover, the independent clinical events committee was in charge of the evaluation of the correctness of the reported end points. The sponsor's biostatistician performed the analyses. The investigators vouch for the completeness and accuracy of the data collection and analysis. One member of the steering committee (M.L.) wrote the report, which was reviewed and approved by all the investigators and the biostatistician.

Center selection was performed to avoid the risk of different expertise levels. ${ }^{8}$ All participating surgeons were consultants trained in both ECC and $\mathrm{OPCAB}$, whose experience was at the plateau of the learning curve for both techniques. Moreover, at each site, randomization was expertisebased, allocating enrolled patients to consultants who at a certain point of their career had decided to use ECC or OPCAB as their prevalent surgical strategy. These surgeons were declared to be experienced operators in OPCAB or ECC with a case load of 50 to 100 CABG operations annually during the previous 5 years.

Patient selection and randomization. All the patients with a euroScore of 6 or more referred for urgent or elective isolated CABG were evaluated for enrollment. The exclusion criteria were age of 18 years or younger, cardiogenic shock requiring emergency surgery and/or preoperative intra-aortic balloon pump, concomitant cardiac or noncardiac surgical procedures, radiographic evidence of a diseased (porcelain) ascending aorta, and an inability or unwillingness of the patient to provide consent. Central randomization assigned the patients in equal proportion to each arm of the study through a computer-generated random number table. Randomization was stratified by center. Eligible patients were randomly assigned to undergo 1 of the 2 treatment options after opening the envelope in the presence of 2 participating consultants ( 1 for ECC and 1 for OPCAB arm) on the day of surgery. Operative planning regarding the number of coronary anastomoses was done before randomization by the 2 consultants surgeons ( 1 for ECC and 1 for OPCAB) assigned to each patient.

A Microsoft Access (Microsoft Office Access 2003; Microsoft, Redmond, Wash) database was used to collect and manage the patients' data. Surgical technique. Standard median sternotomy was the selected surgical access to the heart for all patients. General agreement was reached among the participating centers to perform ECC using normothermia, single crossclamping of the ascending aorta, and cardioplegic arrest and to perform OPCAB after the general principles listed in the K.U. Leuven OPCAB retraining center. ${ }^{9}$ Anticoagulation was obtained using sodium heparin at a dose of $3 \mathrm{mg} / \mathrm{kg}$ for ECC patients and $1.5 \mathrm{mg} / \mathrm{kg}$ for OPCAB patients. Protamine was used at a 1:1 ratio for reversal of the heparin effect. Antifibrinolytic agents were not routinely used. The chosen device for coronary artery stabilization and heart positioning was the Medtronic Octopus and Starfish (Medtronic, Minneapolis, Minn). These devices were not provided free of charge by Medtronic but were bought by each participating center as a part of the routine supply for OPCAB surgery. The type of conduits and harvesting technique, type of cardioplegic solution and method of delivery, type of proximal (aortic or composite) and distal (single or sequential) anastomoses, intraoperative and postoperative management, and the medication regimen were chosen according to local clinical practice.

\section{Study End Points}

The primary postoperative end point was a composite of mortality and major complications comprising myocardial infarction (MI), neurologic complications, renal failure, adult respiratory distress syndrome (ARDS), and reoperation for bleeding, occurring within 30 days after surgery. Operative mortality was defined as death occurring within 30 days of surgery. MI was defined as the appearance of a new $\mathrm{Q}$ wave on the electrocardiogram with creatine kinase-myocardial band isoenzyme fraction increase greater than $100 \mathrm{UI} / \mathrm{L}$ and/or more than $10 \%$ of the total creatine kinase level and/or with documented new wall motion abnormalities other than septal on the echocardiogram. Neurologic complications were defined as stroke (neurologic deficit lasting $>24$ hours with positive computed tomography findings) or transient ischemic attack (neurologic deficit lasting $<24$ hours with positive computed tomography findings). Renal failure was defined as an increased plasma creatinine $\times 2$ associated with urine production less than $0.5 \mathrm{~mL} \cdot \mathrm{kg}^{-1} \cdot \mathrm{h}^{-1} \times 12$ hours. ARDS was defined as the presence of tachypnea (respiratory rate $>30$ breaths $/ \mathrm{min}$ ), bilateral pulmonary infiltrate on chest radiograph, severe hypoxemia (partial pressure of oxygen in arterial blood/fraction of inspired oxygen ratio $<200$ ), need for positive end-expiratory pressure greater than $5 \mathrm{~cm} \mathrm{H}_{2} \mathrm{O}$, no evidence of left ventricular failure (wedge pressure $<18 \mathrm{~mm} \mathrm{Hg}$ ), and no other pathologic features explaining these findings. Reoperation for bleeding was defined as the need for chest reopening in the presence of more than $500 \mathrm{~mL}$ of blood from chest tubes within the first hour, more than $400 \mathrm{~mL}$ within the second hour, more than $300 \mathrm{~mL}$ within the third hour, or total bleeding greater than $1000 \mathrm{~mL}$ within the fourth hour.

\section{Statistical Analysis}

The sample size was estimated, assuming a rate of $20.8 \%$ of the combined end point in the ECC group. ${ }^{10}$ To obtain a reduction of $40 \%$ in the primary end point in the OPCAB group, with a $95 \%$ confidence interval and $80 \%$ power, 630 patients ( 315 per arm) needed to be enrolled. An interim analysis was scheduled at 400 enrolled patients. According to the Pocock method, ${ }^{11}$ the inflation factor to apply to the calculated fixed sample size to preserve $80 \%$ power is 1.1 , with a final sample size of 693 patients. Considering the total significance of $P=.05$, the $\alpha$-spending required at each analysis would be 0.0294 . The categorical data are expressed as the absolute and relative frequency and compared using the chi-square test or Fisher's exact test, as adequate. Continuous data are shown as the mean \pm standard deviation, if Gaussian, and as the median (25th-75th percentiles) otherwise. The skewness of continuous distributions was tested using the Kolmogorov-Smirnov test for 1 sample. Comparisons between normal distributions were performed using the Student $t$ test for independent samples and the nonparametric Mann-Whitney $U$ test in the case of skewed distributions. The primary end point, both composite and split by a single event, was compared using the $\mathrm{Z}$ test or Fisher's exact test, as appropriate. According to the interim nature of the present analysis, $P<.0294$ was considered statistically significant to reject the null hypothesis. 


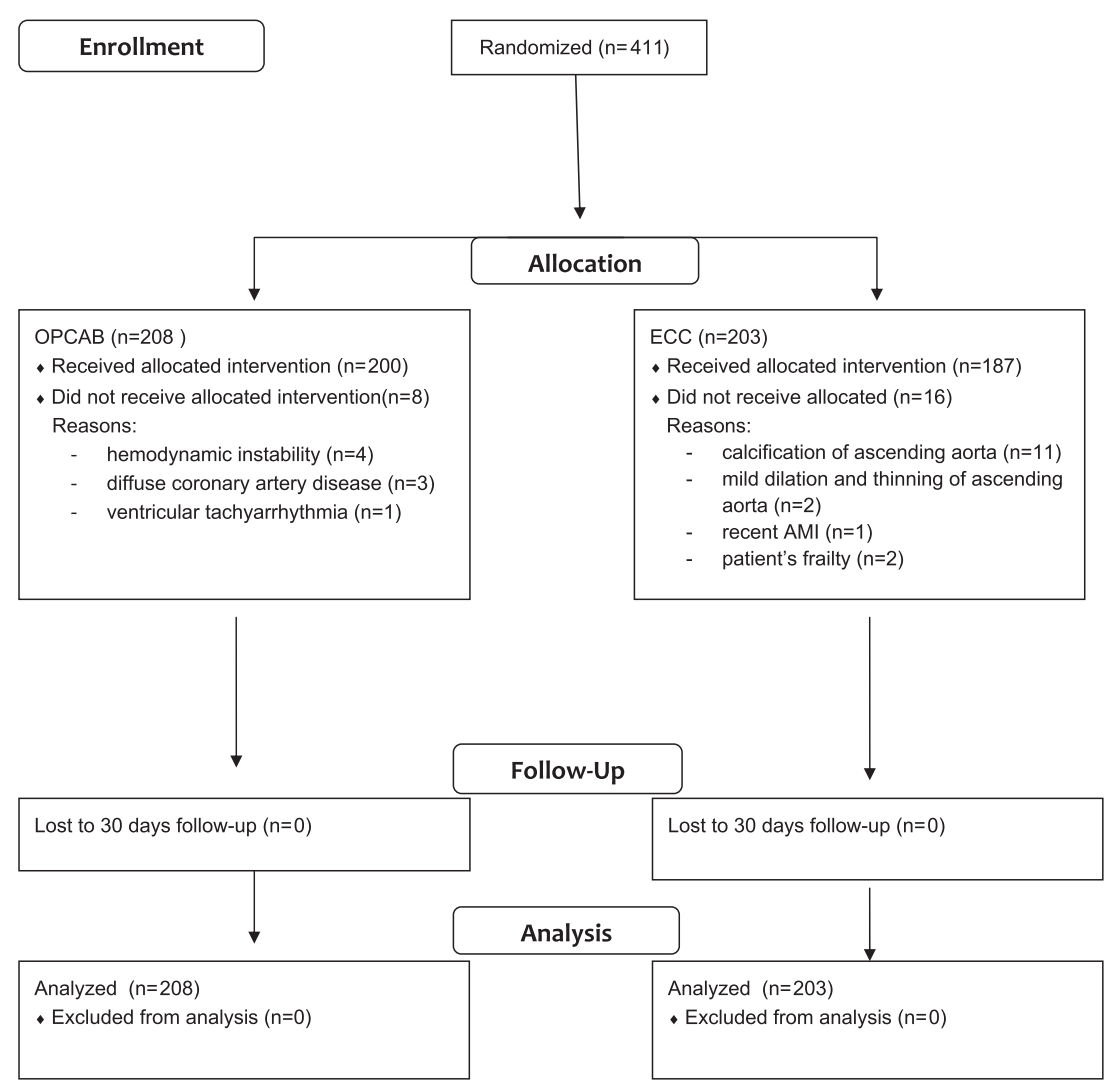

FIGURE 1. Flow diagram of on-off study. $O P C A B$, Off-pump coronary artery bypass; $E C C$, on-pump coronary artery bypass grafting; $A M I$, acute myocardial infarction.

The association between the randomization group and the risk of experiencing the primary end point was evaluated using binary logistic regression analysis. A multivariate model was applied to adjust the results considering the confounding baseline variables, by chance imbalanced in the groups. Discrimination and calibration of the model were evaluated using the area under the curve of the receiver operating characteristic curve and the Hosmer-Lemeshow test, respectively. Randomization was stratified by center, and the possible center-tocenter differences were evaluated applying a generalized mixed model, inserting the center as a fixed effect. As per protocol, all analyses were conducted according to the intention-to-treat principle. $P<.05$ was considered statistically significant, apart from the primary objective.

All analyses were performed using StataSE11 for Windows (StataCorp, College Station, Tex).

\section{RESULTS}

\section{Study Population}

From December 2006 through April 2010, 411 patients were randomly assigned to elective or urgent ECC (203 patients) or OPCAB (208 patients; Figure 1). The baseline demographics and clinical characteristics were well balanced across the 2 groups (Table 1). The mean euroScore was 8 , indicating a population at high surgical risk. OPCAB patients were significantly older $(P=.031)$. The proportion of ECC patients with previous MI was significantly larger $(P=.036)$.

\section{In-Hospital Outcomes}

The number of patients who required conversion to other treatment was double in ECC group $(P=.081$; Table 2). A total of 16 patients $(7.9 \%)$ crossed-over to OPCAB because of intraoperative evidence of an excessively calcified ascending aorta in 11, mild dilation and thinning of the ascending aorta in 2, recent MI in 1, and a decision by the cardiac anesthetist that considered the patient's frailty a contraindication to ECC after randomization in 2. Also, 8 patients $(3.8 \%)$ allocated to OPCAB crossed-over to ECC because of intraoperative hemodynamic instability in 4 , evidence of excessively calcified coronary arteries in 3 , and ventricular tachyarrhythmia in 1 .

The operative time was significantly shorter for the OPCAB group $(P=.004)$. The completeness of the revascularization did not differ significantly between the 2 groups $(P=.241)$, but the mean number of total and venous coronary anastomoses per patient was significantly greater in the ECC group $(P=.001$ and $P<.0001$, respectively), as was the difference between the number of performed and planned distal anastomoses $(P<.0001)$.

\section{Patients' Clinical Outcome}

As reported in Table 3, the intention-to-treat analysis highlighted a significant difference (unadjusted $P=.009$, 
TABLE 1. Preoperative features

\begin{tabular}{|c|c|c|}
\hline Variable & OPCAB $(n=208)$ & $\operatorname{ECC}(n=203)$ \\
\hline Men & $146(70.2)$ & $139(68.5)$ \\
\hline \multicolumn{3}{|l|}{ Age (y) } \\
\hline Mean & 74 & 73 \\
\hline Range & $69-78$ & $68-77$ \\
\hline Current smoker & $48(23.1)$ & $57(28.1)$ \\
\hline Diabetes & $89(42.8)$ & $88(43.3)$ \\
\hline Hypercholesterolemia & $141(67.8)$ & $139(68.5)$ \\
\hline Chronic renal failure & $42(20.2)$ & $28(13.8)$ \\
\hline Hypertension & $174(83.7)$ & $167(82.3)$ \\
\hline Previous CVA & $20(9.6)$ & $20(9.9)$ \\
\hline Peripheral vascular disease & $78(37.5)$ & $71(35.0)$ \\
\hline Carotid stenosis & $78(37.5)$ & 77 (37.9) \\
\hline COPD & $66(31.7)$ & $51(25.1)$ \\
\hline Previous CABG & $1(0.5)$ & $1(0.5)$ \\
\hline Previous AMI & $144(69.2)$ & $159(78.3)$ \\
\hline \multicolumn{3}{|l|}{ NYHA class } \\
\hline I & $46(22.1)$ & $46(22.8)$ \\
\hline II & $101(48.6)$ & $106(52.5)$ \\
\hline III & $56(26.9)$ & $42(20.8)$ \\
\hline IV & $5(2.4)$ & $8(4.0)$ \\
\hline Atrial flutter/fibrillation & $12(5.8)$ & $10(4.9)$ \\
\hline \multicolumn{3}{|l|}{$\mathrm{EF}$} \\
\hline Poor $(<30 \%)$ & $12(5.8)$ & $4(2.0)$ \\
\hline Moderate $(30-50 \%)$ & $106(51.0)$ & $115(56.7)$ \\
\hline Good $(>50 \%)$ & $90(43.3)$ & $84(41.4)$ \\
\hline MR & $120(57.7)$ & $108(53.2)$ \\
\hline Trivial & $95(45.7)$ & $76(37.4)$ \\
\hline Mild & $24(11.5)$ & $30(14.8)$ \\
\hline Moderate & $1(0.5)$ & $2(1.0)$ \\
\hline \multicolumn{3}{|l|}{ euroScore } \\
\hline Mean & 8 & 8 \\
\hline Range & $7-10$ & $7-9$ \\
\hline Unstable angina & $120(57.7)$ & $126(62.1)$ \\
\hline Endovenous antiplatelets & $26(12.5)$ & $22(10.8)$ \\
\hline Endovenous nitrates & $39(18.8)$ & $50(24.6)$ \\
\hline Left main disease & $79(38.0)$ & $83(40.9)$ \\
\hline Mean vessel disease & $2.6(0.6)$ & $2.6(0.6)$ \\
\hline
\end{tabular}

Data presented as number of patients, with percentages in parentheses, unless otherwise noted. $O P C A B$, Off-pump coronary artery bypass; $E C C$, on-pump coronary artery bypass; $C V A$, cerebrovascular accident; $C O P D$, chronic obstructive pulmonary disease; $C A B G$, coronary artery bypass graft; $A M I$, acute myocardial infarction; $N Y H A$, New York Heart Association; $E F$, ejection fraction; $M R$, mitral regurgitation; euroScore, European System for Cardiac Operative Risk Evaluation.

adjusted $P=.010$ ) in the rate of the primary postoperative end point between the ECC $(13.3 \%)$ and OPCAB $(5.8 \%)$ groups. Although not statistically significant, all the components of the composite end point (operative mortality, MI, neurologic complications, renal failure, reoperation for bleeding) but 1 (ARDS) favored OPCAB patients. The risk to experience the primary end point was significantly greater in the ECC than in the OPCAB group (unadjusted odds ratio, 2.51; 95\% confidence interval, 1.23-5.10; $P=.011$; adjusted odds ratio, $3.07 ; 95 \%$ confidence interval, $1.32-7.14 ; P=.009)$. No differences were found among the centers $(P=.383)$.
TABLE 2. Operative results

\begin{tabular}{|c|c|c|c|}
\hline Variable & $\begin{array}{c}\text { OPCAB } \\
(n=208)\end{array}$ & $\begin{array}{c}\text { ECC } \\
(n=203)\end{array}$ & $\begin{array}{c}P \\
\text { value }\end{array}$ \\
\hline Crossover & $8(3.8)$ & $16(7.9)$ & .081 \\
\hline Procedure time $(\mathrm{min})$ & $226 \pm 59$ & $242 \pm 58$ & .004 \\
\hline Mean number of total anastomoses & $3.0 \pm 1.1$ & $3.3 \pm 1.0$ & .001 \\
\hline Mean number of arterial anastomoses & $1.7 \pm 0.9$ & $1.6 \pm 0.8$ & .402 \\
\hline Mean number of venous anastomoses & $1.5 \pm 1.0$ & $2.0 \pm 1.0$ & $<.0001$ \\
\hline Coronary anastomoses (n) & & & .010 \\
\hline 1 & $13(6.3)$ & $5(2.5)$ & \\
\hline 2 & $57(27.4)$ & 38 (18.7) & \\
\hline 3 & $86(41.3)$ & 83 (40.9) & \\
\hline 4 & $39(18.8)$ & $54(26.6)$ & \\
\hline 5 & $11(5.3)$ & $21(10.3)$ & \\
\hline 6 & 0 & $2(1.0)$ & \\
\hline 9 & $2(1.0)$ & 0 & \\
\hline $\begin{array}{l}\text { Performed vs planned distal } \\
\text { anastomoses (n) }\end{array}$ & & & $<.0001$ \\
\hline Mean & 0 & 1 & \\
\hline Range & $0-1$ & $0-1$ & \\
\hline Composite graft & $69(33.2)$ & $44(21.7)$ & .009 \\
\hline ITA graft (n) & & & .335 \\
\hline 0 & $2(1)$ & $6(3)$ & \\
\hline 1 & $173(83.2)$ & $167(82.3)$ & \\
\hline 2 & $33(15.9)$ & $30(14.8)$ & \\
\hline RA graft (n) & & & .807 \\
\hline 0 & $165(79.3)$ & $163(80.3)$ & \\
\hline 1 & $43(20.7)$ & $40(19.7)$ & \\
\hline 2 & 0 & 0 & \\
\hline Endarterectomy & $5(2.4)$ & $4(2.0)$ & .764 \\
\hline Incomplete revascularization & $20(9.6)$ & $27(13.3)$ & .241 \\
\hline CPB time (min) & & $83 \pm 58$ & \\
\hline ACC time (min) & & $55 \pm 35$ & \\
\hline Coronary shunt & $187(89.9)$ & & \\
\hline Heart positioner & $143(67.8)$ & & \\
\hline
\end{tabular}

Data presented as number of patients, with percentages in parentheses, unless otherwise noted. $O P C A B$, Off-pump coronary artery bypass; $E C C$, on-pump coronary artery bypass; ITA, internal thoracic artery; $R A$, radial artery; $C P B$, cardiopulmonarybypass; $A C C$, aortic crossclamp.

\section{DISCUSSION \\ Principal Findings}

The on-off study is the first RCT comparing ECC and OPCAB in high-risk patients (euroScore of $\geq 6$ ) demonstrating the superiority of OPCAB compared with ECC in the postoperative combined end point (operative mortality, MI, neurologic complications, renal failure, reoperation for bleeding).

\section{Comparison With Other Studies}

The present on-off study did not confirm the results of other recently published RCTs that included both with low-risk ${ }^{12}$ and high-risk ${ }^{13}$ patients. The main difference between our study and the cited RCTs was that the present study was an expertise-based trial, run by consultants working in group practices. The surgeons were well qualified, at the plateau of their learning curve, had a well-documented 
TABLE 3. Primary end point

\begin{tabular}{lcccc}
\hline \multicolumn{1}{c}{ End point } & $\begin{array}{c}\text { ECC } \\
(\mathbf{n}=\mathbf{2 0 3})\end{array}$ & $\begin{array}{c}\text { OPCAB } \\
(\mathbf{n}=\mathbf{2 0 8})\end{array}$ & $\begin{array}{c}\text { Unadjusted } \\
\boldsymbol{P} \text { value }\end{array}$ & $\begin{array}{c}\text { Adjusted } \\
\boldsymbol{P} \text { value }\end{array}$ \\
\hline Composite & $27(13.3)$ & $12(5.8)$ & .009 & $.010^{*}$ \\
Operative mortality & $7(3.4)$ & $4(1.9)$ & .376 & .379 \\
Myocardial infarction & $6(3.0)$ & $4(1.9)$ & .539 & .514 \\
Stroke & $1(0.5)$ & - & .494 & .995 \\
Renal failure & $10(4.9)$ & $5(2.4)$ & .173 & .149 \\
Reoperation for bleeding & $7(3.4)$ & $3(1.4)$ & .216 & .115 \\
ARDS & - & $1(0.5)$ & 1.000 & .995 \\
\hline
\end{tabular}

Data presented as number of patients, with percentages in parentheses. OPCAB, Offpump coronary artery bypass; $E C C$, on-pump coronary artery bypass; $A R D S$, adult respiratory distress syndrome. ${ }^{*}$ Hosmer-Lemeshow $=0.978$; area under the curve, $0.64 ; 95 \%$ confidence interval, $0.56-0.73 ; P=.003$.

surgical practice, and performed only the procedure they preferred, in which they had expertise and that they used routinely during their daily practice (ECC or OPCAB). In the Randomized On/Off Bypass trial, ${ }^{12}$ some of the participating surgeons were trainees and were required to document at least $20 \mathrm{OPCAB}$ procedures to participate in the study. In general, the success of a surgical techniques improves with much more extensive experience than that required in the Randomized On/Off Bypass trial. An observational study of 5678 isolated CABG procedures ${ }^{14}$ showed a progressive decrease in the observed and adjusted-risk mortality during the first 4 years of independent practice. Considering that $\mathrm{OPCAB}$ is recognized as more technically demanding than ECC, we can argue that in the Randomized On/Off Bypass trial, surgeons could not have been adequately trained. This has also been witnessed by the high number of procedural crossovers: $137(12.4 \%)$ in the OPCAB group and $40(3.6 \%)$ in the ECC group $(P<.001)$. In contrast, during the present on-off study, the number of procedural crossovers was $8(3.8 \%)$ in the OPCAB group and $16(7.9 \%)$ in the ECC group $(P=.081)$. The Best Bypass Surgery (BBS) trial $^{13}$ better addressed the issue of the lack of expertise. In that single-center study, OPCAB had been performed routinely for 2 years before the launch of the trial and each of the 3 participating surgeons performed at least $50 \%$ of their $\mathrm{CABG}$ procedures using OPCAB. However, it was not designed as an expertise-based trial (the 3 participating surgeons performed both ECC and OP$\mathrm{CAB}$ during the study), and the surgeons were aware of which procedures they performed and could have unconsciously biased the trial findings, favoring the procedure they believed might give better outcomes. This was not possible during the present on-off study, because surgeons could only be unconsciously biased toward the procedure they preferred, in which they had greater experience, and which they used during both the trial and their daily practice.

\section{Surgical Procedure and Outcome}

In the present on-off study, there was a remarkably low rate of neurologic events in both the ECC $(0.5 \%)$ and
OPCAB $(0 \%)$ groups $(P=.494)$. This finding can be related to the relatively frequent use of composite grafts (21.7\% in the ECC group and $33.2 \%$ in the OPCAB group, $P=.009$ ), with single clamping of the ascending aorta in ECC patients and a $7.9 \%(n=16)$ conversion rate from ECC to OPCAB $(n=11)$ for a severely diseased ascending aorta. All these factors contributed to reducing ascending aorta manipulation. The absence of neurologic events in OPCAB patients and the low rate of procedural conversion to ECC are testament to the hemodynamic stability during OPCAB. The use of coronary shunts $(89.9 \%)$ and a heart positioner $(67.8 \%)$ was fundamental in achieving this result. Hemodynamic stability and the avoidance of cardiopulmonary bypass with nonpulsatile flow could also explain the lower incidence of postoperative renal failure in the OPCAB group ( $2.4 \%$ vs $4.9 \%, P=.173)$. Reoperation for bleeding was lower, but the difference was not statistically significant, in the OPCAB group. In contrast, the incidence of blood transfusion was significantly greater among ECC patients $(65.5 \%$ vs $50.5 \%, P=.002)$. The procedure time was significantly shorter in the OPCAB group $(P=.004)$ but with a significantly lower mean number of distal anastomoses per patient $(P=.001)$. However, the incompleteness of revascularization (determined by the number of grafts planned vs the number performed) was almost identical $(P=.241)$, as was the mean number of coronary vessel disease (Table 1). A possible explanation is that during ECC, the surgeons decided to perform bypass on coronary arteries they had not planned preoperatively, such as was shown by the significant difference between the number of performed and planned distal anastomoses $(P<.0001)$.

\section{Implications}

Coronary patients tend to be older and more ill, with high frailty index, a high predicted risk of mortality, and complex 3 -vessel coronary artery disease. This picture is peculiar to the group of patients requiring surgical treatment and who will be a part of the practice of cardiac surgeons in the coming years. Because they are fragile, high-risk patients, their surgical treatment should be less stressful for the whole body. In the United States, there is already a national trend ${ }^{15}$ that favors OPCAB for elderly and frail patients, in the belief that cardiopulmonary bypass could be dangerous for them. This trend is based on the results from several retrospective analyses of large databases that have clearly shown the reduced risk-adjusted mortality after OPCAB compared with after ECC. ${ }^{16,17}$ The present on-off study has corroborated and completed the results of these studies. It is the first RCT to show OPCAB to be safer than ECC at 30 days for high-risk patients. The primary composite end point (operative mortality, MI, neurologic complications, renal failure, reoperation for bleeding) favored OPCAB compared with ECC in the intention-to-treat analysis (unadjusted $P=.009$, adjusted $P=.010$ ). A trend was found toward 
reduced crossovers in the OPCAB group $(3.8 \%$ vs $7.9 \%$, $P=.081$ ), because of the study design that reduced procedural crossovers resulting from technical problems. The greater rate of conversion to other treatment in the ECC group was not related to the surgical technique but mainly to anatomic reasons, such as a severely diseased ascending aorta, a frequent finding in this patient population.

\section{Study Limitations}

The present on-off was a sponsored study. However, to reduce the risk of bias, the outcomes were assessed by an independent clinical events committee composed of 3 physicians with a relevant medical background in the field of cardiac surgery (who were unaware of the allocated treatment). The sponsor's biostatistician performed blinded analyses of the primary end point, receiving data with a coded indication of the randomized group assigned to each patient.

\section{CONCLUSIONS}

In high-risk patients (euroScore of $\geq 6$ ), OPCAB reduces early mortality and morbidity (MI, neurologic complications, renal failure, reoperation for bleeding, ARDS) compared with ECC.

\section{References}

1. Weiland AP, Walker WE. Physiologic principles and clinical sequelae of cardioplulmonary bypass. Heart Lung. 1986;15:34-9.

2. Edmunds LH Jr. Inflammatory response to cardiopulmonary bypass. Ann Thorac Surg. 1998;66:S12-6

3. Nathoe HM, van Dijk D, Jansen EW, Suyker WJ, Diephuis JC, van Boven WJ, et al. A comparison of on-pump and off-pump coronary bypass surgery in lowrisk patients. $N$ Engl J Med. 2003;348:394-402.

4. Moller CH, Penninga L, Wetterslev J, Steinbruchel DA, Gludd C. Clinical outcomes in randomized trials of off- vs on-pump coronary artery bypass surgery: systematic review with meta-analyses and trial sequential analyses. Eur Heart J. 2008;29:2601-16.

5. Stamou SC, Jablonski KA, Hill PC, Bafi AS, Boyce SW, Corso PJ. Coronary revascularization without cardiopulmonary bypass versus the conventional approach in high-risk patients. Ann Thorac Surg. 2005;79:552-7.

6. Puskas JD, Thourani VH, Kilgo P, Cooper W, Vassiliades T, Vega D, et al. Offpump coronary artery bypass disproportionately benefits high-risk patients. Ann Thorac Surg. 2009;88:1142-7.

7. Moller C, Perko MJ, Lundt JT, Andersen LW, Kelbaek H, et al. No major differences in 30-day outcomes in high-risk patients randomized to off-pump versus on-pump coronary bypass surgery. Circulation. 2010;121:498-504.

8. Devereaux PJ, Bhandari M, Clarke M, Montori VM, Cook DJ, Yusuf S, et al. Need for expertise based randomized controlled trials. BMJ. 2005;330:88.

9. K.U. Leuven. The K.U. Leuven OPCAB re-training centre: concepts and experience in the re-training off-pump coronary artery bypass surgery, June 2005. Available at: http://www.opcab-training.eu. Accessed Dec 2011.

10. Al-Ruzzeh S, Nakamura K, Athanasiou T, Modine T, George S, Yacoub M, et al. Does off-pump coronary artery bypass (OPCAB) surgery improve the outcome in high-risk patients?: a comparative study of 1398 high-risk patients. Eur J Cardiothorac Surg. 2003;23:50-5.

11. Pocock SJ. Group sequential methods in the design and analysis of clinical trials. Biometrika. 1977;64:191-9.

12. Shroyer AL, Grover FL, Hattler B, Collins JF, McDonald GO, Kozora E, et al. On-pump versus off-pump coronary artery bypass surgery. $N$ Engl $\mathrm{J} \mathrm{Med}$. 2009;361:1827-37.

13. Møller CH, Perko MJ, Lund JT, Andersen LW, Kelbæk H, Madsen JK, et al. No major differences in 30-day outcomes in high-risk patients randomized to offpump versus on-pump coronary artery bypass surgery: the Best Bypass Surgery trial. Circulation. 2010;121:498-504.
14. Bridgewater B, Grayson AD, Au J, Hassan R, Dihmis WC, Munsch C, et al. Improving mortality of coronary surgery over first four years of independent practice: retrospective examination of prospectively collected data from 15 surgeons. BMJ. 2004;329:421.

15. Puskas JD, Edwards FH, Pappas PA, O'Brien S, Peterson ED, Kilgo P, et al. Offpump techniques benefit men and women and narrow the disparity in mortality after coronary bypass grafting. Ann Thorac Surg. 2007;84:1447-56.

16. Plomondon ME, Cleveland JC, Ludwig ST. Off-pump coronary artery bypass is associated with improved risk-adjusted outcomes. Ann Thorac Surg. 2001;72:114-9.

17. Puskas DJ, Thourani VH, Kilgo P, Cooper W, Thomas V, Vega JD, et al. Offpump coronary artery bypass disproportionately benefits high-risk patients. Ann Thorac Surg. 2009;88:1142-7.

\section{Discussion}

Dr Joseph F. Sabik (Cleveland, Ohio). I would like to congratulate Dr Lemma and colleagues on a fine presentation and study and thank them for providing me with a copy of their manuscript and presentation in advance of the meeting.

I have 3 questions. In this study, 411 patients were entered, however, you do not tell us how many patients were screened and, of those screened, what percentage were eligible and, of those eligible, what percentage were actually enrolled in the study. This information is important in deciding what percentage of patients undergoing coronary surgery this study is applicable to and to whether it is representative of most patients with a euroScore of 6 or higher. Can you provide this information?

My second question is, the study was designed as an expertisebased randomized controlled trial. In other words, as you explained, if a patient was randomized to off-pump surgery, his surgery was performed by an expert in off-pump surgery, and if the patient was randomized to on-pump surgery, his surgery was performed by an expert in on-pump surgery. Therefore, in addition to being randomized to 2 different procedures, the patients were randomized to 2 different groups of surgeons. How can we determine whether the better outcomes observed in the off-pump patients were because of off-pump surgery being better or the possibility that the off-pump surgeons were better surgeons and therefore their patients did better?

My final question has to do with revascularization. Similar to other studies comparing on- and off-pump surgery, fewer bypass grafts were performed in the off-pump surgery group. This is particularly alarming, because this study is an expertise-based study. This suggests that off-pump surgery, even in expert hands, might be a less-effective revascularization strategy than on-pump surgery. Understanding the importance of the completeness of revascularization on long-term outcomes, do the better early outcomes in off-pump surgery justify the potential long-term risk?

Thank you very much.

Dr Lemma. Thank you very much, Dr Sabik, for your comments. About your first question, we do not know precisely how many patients were screened and how many were eligible as a direct consequence of the study design. The on-off study was an expertise-based trial run by consultants working in group practices. This means that we had plenty of screened and eligible patients but only a few of them could be randomized because the study protocol required the simultaneous presence of 2 consultants for the same patient, 1 an expert in off-pump and 1 an expert in onpump surgery at the moment of envelope opening in the operating room. In a few hospitals, these data were not always recorded so I can't give you figures. This is also the reason the study took roughly 4 years to be completed. 
Dr Sabik. Can I ask a follow-up. Do you have an idea of what percentage of patients undergoing surgery have a euroScore greater than 6 ? Is it $5 \%, 10 \%, 20 \%$ ?

Dr Lemma. I don't know precisely the figure how many patients had a euroScore greater than 6 among the screened and eligible patients. I can tell you that the average euroScore was 8 in both groups, on-pump and off-pump patients.

About the question related to the number of distal anastomoses, this is an interesting point in the sense that we had 3.0 distal anastomoses in the off-pump group and 3.3 in the on-pump group. However, if you consider the difference between the planned versus performed distal anastomoses, in the off-pump group, the number of distal anastomoses performed was equal to the number of distal anastomoses predicted, but in the on-pump group, the number of distal anastomoses performed was greater than the number of distal anastomoses predicted. Thus, it seems that there was an attitude in the on-pump group to perform more distal anastomoses than predicted before the operation.

And the last question was?

Dr Sabik. Something we don't often see in surgery is an expertise-based trial. It obviously adds the confounder of different surgical groups.

Dr Lemma. Well, I would see the problem the other way around in the sense that expertise-based studies are important because only an expert surgeon can precisely apply a given surgical technique. Moreover, in an expertise-based study, you do not have a surgeons who can be consciously or unconsciously biased toward a treatment, because they do believe the treatment they apply is the best. If you consider other prospective randomized trials, such as the Best Bypass Surgery study published recently in the journal Circulation, they enrolled 3 surgeons who performed both onpump and off-pump surgery. However, we can't imagine that they were neutral toward these 2 procedures. For sure, they preferred 1 of these 2, and so they could have consciously or unconsciously been biased toward 1 of these 2 treatments. In our study, the surgeons couldn't be biased, either consciously or unconsciously, toward on-pump or off-pump surgery because they believed the treatment they applied was the best one.

Dr Sabik. Thank you.

Dr Lokeswara Rao Sajja (Hyderabad, India). I congratulate Dr Lemma for an excellently designed and executed study. I have a couple of questions. In your study, was the preoperative serum creatinine level comparable between the 2 groups? Because even a marginal increase in the preoperative creatinine in 1 group can lead to an increased incidence of postoperative renal failure in that group. I also find that in the intention-to-treat analysis, there was no significant difference in the incidence of renal failure between the ECC group and the OPCAB group, but the in on-treatment analysis, there was a significant difference. Is this difference due to the conversion of the cases from OPCAB to ECC?

Dr Lemma. Thank you for your question. There was no difference between the 2 groups in the renal function. The only differences among the preoperative clinical characteristics were in age-we had older patients in the off-pump group — and myocardial infarction, of which we had a greater incidence in the on-pump group.

After on-treatment analysis, which means considering crossovers, renal function became statistically significant, per se. This probably means that the hemodynamic stability of off-pump surgery was ideal to preserve renal function, but in the on-pump group, the continuous flow due to the pump probably decreased the function of the patient with preoperative borderline renal function.

Dr Sajja. It is presented that reoperation for postoperative bleeding was significantly greater in the ECC group. Was the protocol to stop antiplatelet drug therapy uniform in both the groups? Were the patients on single antiplatelet or dual-antiplatelet therapy before the patients were subjected to surgery?

Dr Lemma. The antiplatelet protocol was the same in both groups. The patients usually stopped antiplatelets at least 5 days before surgery.

Dr John D. Puskas (Atlanta, Ga). Dr Lemma, I congratulate you on a landmark study in off-pump bypass, a subject near and dear to my own heart. It is interesting to see this expertise-based randomization strategy. I do believe it is an important way to look at this problem of comparing off-pump and on-pump bypass. Certainly, the failure to do that sort of equivalence of expertise in both surgical groups in the Randomized On/Off Bypass trial was a fundamental flaw of that trial, and you have avoided that with your design. I have 3 questions.

You commented on the completeness of revascularization, that is, the number of grafts performed versus planned having been different between the groups. Was the completeness of revascularization accomplished in the lateral wall in the off-pump group? That is where the rubber hits the road. Were your surgeons able to graft the lateral wall effectively and completely in the off-pump group? Was the extra graft or 0.3 of a graft in the on-pump group sort of a gratuitous extra diagonal graft by a motivated team of onpump surgeons?

The second question, was the use of arterial conduits similar between the groups?

Finally, can you share with us any information about the length of stay or cost comparisons between these 2 groups?

Again, congratulations on a terrific study.

Dr Lemma. Well, I don't have an answer for your last question in the sense that a cost-comparison analysis was not planned in the study design, and the length of stay was a part of secondary end point analysis. Thus, these data will be analyzed in conjunction with 1 year of follow-up. About the question of whether in the off-pump group, also the obtuse marginal branch was easily bypassed, the answer is yes. If you consider the data, $70 \%$ of surgeons routinely used a heart positioner and $90 \%$ routinely used intracoronary shunts. This means that according to the principles espoused by Paul Sergeant in his Web site related to the teachable components of off-pump surgery, the surgeons were able to maintain a perfect hemodynamic stability throughout the operation without any problem bypassing the obtuse marginal branch.

And the second question, Dr Puskas, was?

Dr Puskas. Arterial grafting. Was the use of arterial conduits similar between the groups and what was the use of arterial conduits?

Dr Lemma. No difference was found between the use of arterial conduits in the 2 groups, neither as number nor as type. The only difference was in the number of venous grafts, it was greater in the on-pump group, as you said probably a sort of gratuitous extra graft by a motivated team of on-pump surgeons.

Dr Puskas. Thank you. 